\title{
Fetal and infantile hypertension caused by unilateral renal arterial disease
}

\author{
D I Wilson, R E Appleton, M G Coulthard, R E J Lee, C Wren, H H Bain
}

\begin{abstract}
Three children who presented with heart failure in infancy caused by severe hypertension as a result of unilateral renal arterial disease are described. One presented at 3 days of age with persistent fetal circulation and heart failure. He had abnormal great vessels that indicated that the hypertension was of long standing and therefore fetal; this has not been described previously. The other two children failed to thrive because of unrecognised hypertension and subsequently presented with heart failure. All three underwent unilateral nephrectomy which cured their hypertension, and all were thriving at the time of writing. The benefits of nephrectomy outweighed the operative risks and loss of renal function. Blood pressure should be measured in children who are failing to thrive as part of routine clinical practice.
\end{abstract}

Hypertension in the neonatal period and early infancy is underdiagnosed. We describe here three children with hypertension associated with unilateral renal arterial disease all of whom presented with life threatening cardiac failure. All three were successfully treated by unilateral nephrectomy. In one patient the hypertension had developed in utero, a finding that has not to our knowledge been previously reported.

\section{Case reports}

CASE 1

This boy was born at 38 weeks' gestation weighing $3980 \mathrm{~g}$ to a 26 year old mother who had insulin dependent diabetes mellitus. There was no relevant family history, and the pregnancy had been normal. Delivery was by Neville Barnes forceps and was complicated by a left Erb's palsy and fractures of the left clavicle and left sixth rib. No resuscitation was required.

Department of Child

Health, Sir James Spence Building, Royal Victoria Infirmary,

Newcastle upon Tyne

NE1 4LP

D I Wilson

R E Appleton

M G Coulthard

R E J Lee

Department of Paediatric Cardiology, Freeman Hospital,

Newcastle upon Tyne C Wren

H H Bain

Correspondence to: Dr Coulthard.

Accepted 28 March 1990 from 75 to $95 \%$ when he was given an infusion of tolazoline. On day 5 he developed increasing respiratory distress and required ventilation. Twenty four hours later his systolic blood pressure increased from $90 \mathrm{~mm} \mathrm{Hg}$ to 145 mm $\mathrm{Hg}$, confirmed by Doppler measurement, auscultation, and palpation. Intravenous hydralazine given by infusion controlled his blood pressure, but 12 hours later an intravenous infusion of labetalol was also required. Both infusions were discontinued after five days. Thirty six hours later the systolic blood pressure increased to $100 \mathrm{~mm} \mathrm{Hg}$, which responded to propranolol and hydralazine given orally.

Results of the following investigations were normal: full blood count; plasma concentrations of urea, creatinine, electrolytes, calcium, and phosphate; alkaline phosphatase and cardiac enzyme activities; concentrations of thyroxine, cortisol, and $17 \alpha$ hydroxyprogesterone; and urinary 4-hydroxy-3-methoxy mandelic acid (HMMA):creatinine and homovanillic acid (HVA):creatinine ratios. Antibodies (acute and convalescent) to Coxsackie, herpes, rubella, cytomegalovirus, mumps, and adenovirus were not detected in serum.

Renal ultrasonography showed no abnormality. A dimercaptosuccinic acid nucleide (DMSA) scan showed that the left kidney contributed less than a quarter of the total renal function. An abdominal aortogram showed a normal right kidney with a normal vascular tree. The left main renal artery was normal, but the intrarenal arteries were abnormal with leashes of smaller vessels. A left selective renal arteriogram showed a patchy nephrogram with some areas of dense uptake and others with almost none (fig 1). It was not possible to carry out a right selective renal arteriogram. A thoracic aortogram showed pronounced dilatation of the aortic arch (fig 2). The carotid arteries were dilated and tortuous, as were the subclavian vessels, although these tapered into brachial arteries of normal diameter. Plasma renin activities in the left renal vein, inferior vena cava, and right atrium were raised at $7 \cdot 5-8 \cdot 6$ $\mathrm{ng} / \mathrm{l} / \mathrm{sec}$ (27 000-31 $000 \mathrm{ng} / \mathrm{l} / \mathrm{hour}$ ) (upper limit of reference range for age is $2.5(9100)$ ). The right renal vein could not be sampled.

Plasma renin activity must be interpreted with caution in patients receiving hypotensive drugs or under general anaesthesia and the principal value of sampling the renal vein is for comparison of the two sides. ${ }^{1}$ With such high activities, together with the radiological asymmetry, however, it was concluded that the infant had renin driven hypertension, probably 


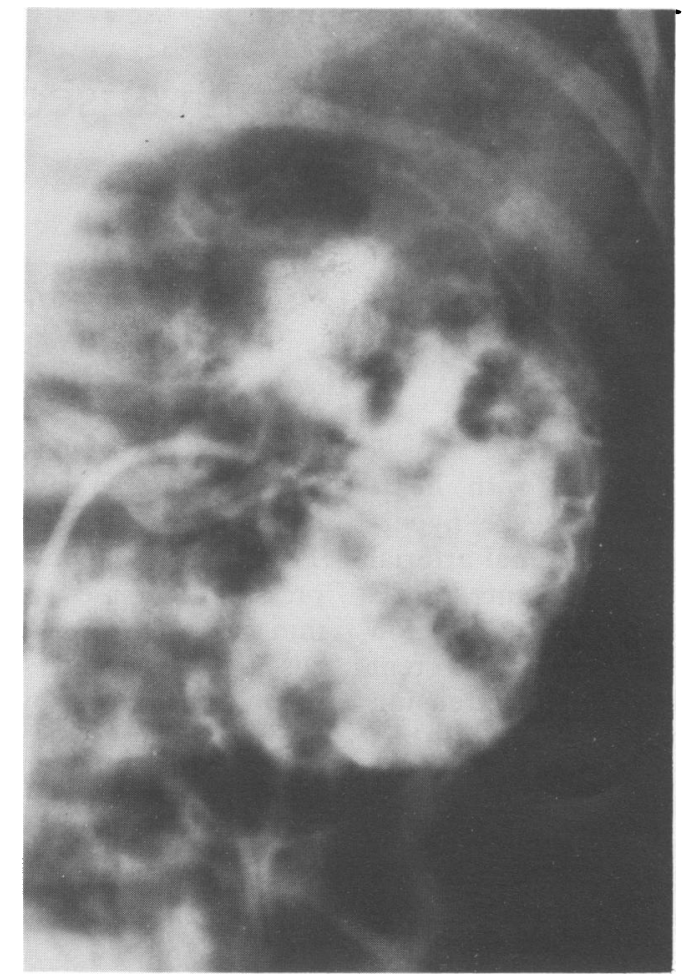

Figure 1 Left renal arteriogram in the venous phase showing a patchy nephrogram with some areas of dense uptake and others with almost no uptake.

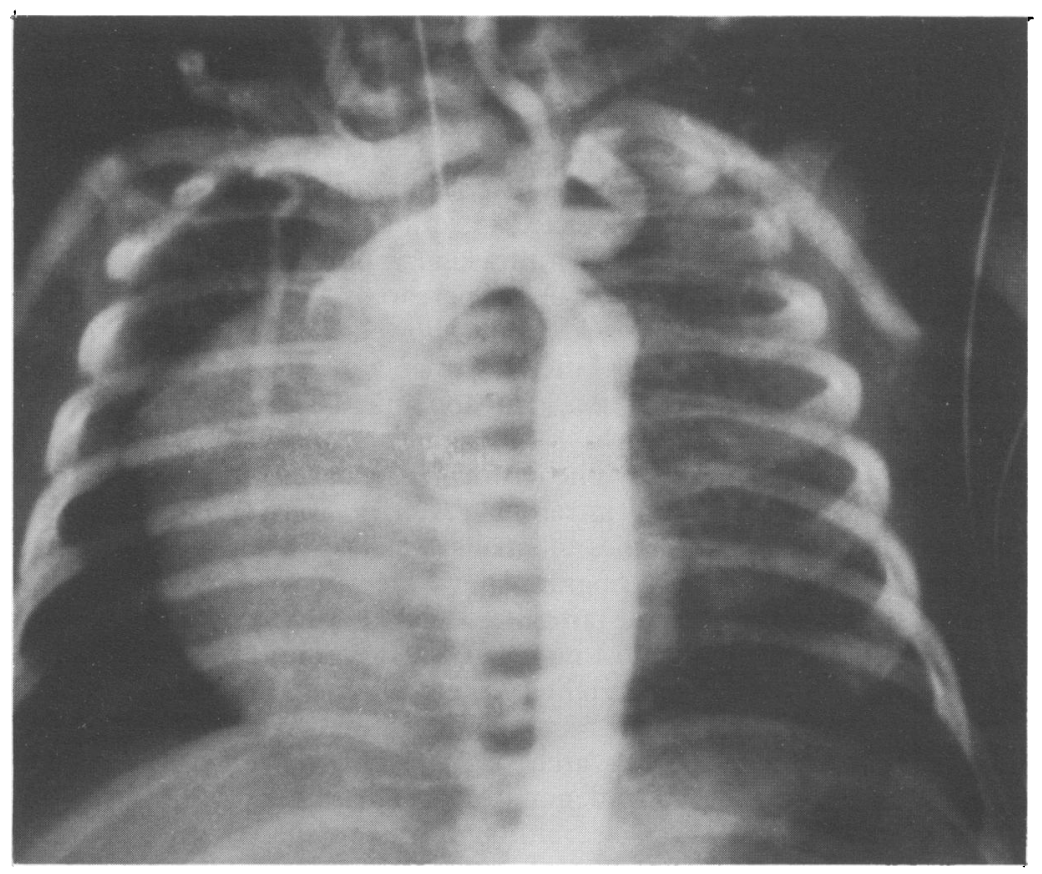

Figure 2 Thoracic arteriogram showing a grossly widened aorta and tortuous and dilated carotid and subclavian arteries.

caused by the abnormal left kidney. He was treated with hydralazine and propranolol. A left nephrectomy was carried out without complications when he was 3 months old, and thereafter the systolic blood pressure remained between 70 and $80 \mathrm{~mm} \mathrm{Hg}$ without treatment. At 17 months of age physical examination showed no abnormality, and echocardiography and peripheral plasma renin activity were normal.
Histological examination of the left kidney showed patchy areas of abnormal cortex with extensive fibrosis and a chronic inflammatory cell infiltrate with deposits of haemosiderin, indicating previous haemorrhage. Loss of tubules (mainly proximal) was evident, and many of the surviving tubules seemed immature and contained hyaline casts. Arteries within the damaged cortical areas were thick walled but not characteristic of fibromuscular dysplasia, and the vessels at the hilum were normal. There was no evidence of renal dysplasia.

\section{CASE 2}

This boy was born at full term weighing $3300 \mathrm{~g}$ after a normal pregnancy and delivery. There was no relevant family history. The perinatal period was normal and he was well until 12 months of age when he presented with failure to thrive. No cause was identified, but his blood pressure was not measured. Weight gain remainded poor and he became progressively more lethargic. On admission to hospital at the age of 2.3 years he had both left and right heart failure. The blood pressure in both arms was 144/95 mm Hg by Doppler measurement and auscultation. His weight was below the third centile. Chest radiography showed cardiomegaly and pulmonary oedema. Electrocardiography showed sinus tachycardia, and right atrial and left ventricular hypertrophy. Cross sectional echocardiography showed that the left ventricle was dilated and hypertrophied, and contracting poorly together with regurgitation through the mitral and tricuspid valves and hypertrophy of the papillary muscles.

Results of the following investigations were normal: full blood count; plasma concentrations of creatinine and electrolytes, calcium, phosphate and immunoglobulins; tests of thyroid function; cardiac enzyme activities, 24 hour urinary HVA and HMMA excretion, and urine culture. Intravenous urography showed a small left kidney with a normal caliceal appearance and normal excretion. The right kidney was hypertrophied. A DMSA isotope scan showed that the left kidney contributed less than $15 \%$ of total renal function. Renal arteriography showed complete occlusion of the left main renal artery. The left kidney was small and supplied by a tortuous vessel from the aorta. The right kidney was enlarged and had a normal vascular tree. Plasma renin activities from both renal veins were increased to greater than $26.7 \mathrm{ng} / \mathrm{l} / \mathrm{sec}$ (96 $000 \mathrm{ng} / \mathrm{l} /$ hour) (upper limit of reference range for age is $0.7(2610))$. The samples were insufficient to repeat the assay at dilutions, so that any discrepancy between the two sides could not be measured.

He was treated with digoxin, spironolactone, chlorothiazide, and hydralazine and his cardiac failure improved. His blood pressure remained raised at $150 / 95 \mathrm{~mm} \mathbf{H g}$. After the addition of propranolol and captopril to his regimen, the blood pressure fell to $100 / 45 \mathrm{~mm} \mathrm{Hg}$.

The above investigations indicated that the patient's heart failure was secondary to renovascular induced hypertension as a result of stenosis of the main left renal artery. A left 
nephrectomy was performed when he was $2 \cdot 5$ years old. Histological examination of the kidney showed no evidence of renal artery dysplasia. His postoperative recovery was uncomplicated and antihypertensive drugs were withdrawn over the following three months. At 4 years of age he was well with a normal blood pressure $(105 / 50 \mathrm{~mm} \mathrm{Hg}$ ), and physical examination showed no abnormality. His height and weight were on the 25th centile. Echocardiography and the peripheral plasma renin activity were normal.

\section{CASE 3}

This girl was born at full term weighing $3200 \mathrm{~g}$ after a normal pregnancy. She was well until 10 months of age when she developed gastroenteritis. On admission to a local hospital she was seriously ill: her weight was below the third centile, she had poor peripheral perfusion, and a systemic blood pressure of $70 / 40 \mathrm{~mm} \mathrm{Hg}$. She required resuscitation with an intravenous infusion of plasma but immediately developed severe right and left heart failure. She was transferred to this hospital for ventilatory support. After resuscitation with dobutamine and sodium bicarbonate her blood pressure rose to $160 / 100 \mathrm{~mm} \mathrm{Hg}$, and remained at that level. Ventilatory support was withdrawn after five days. Blood pressure control was difficult and she required nitroprusside and labetalol intravenously, and subsequently propranolol and hydralazine orally.

Respiratory syncytial virus was isolated from tracheal secretions. Cultures of blood, urine, and cerebrospinal fluid were sterile and paired serum viral antibody titres excluded any recent infection. Chest radiography showed pulmonary oedema and left atrial enlargement. Cross sectional echocardiography showed a dilated left ventricle with poor contractility and mitral and tricuspid regurgitation. A renal ultrasound scan showed a small right, and an enlarged left, kidney. A DMSA isotope scan showed that the right kidney contributed $23 \%$ of total renal function. Renal arteriography showed severe stenosis of the main right renal artery and a normal left side. Peripheral plasma renin activity (with the patient conscious but receiving hypotensive drugs), was raised at $4.9 \mathrm{ng} / \mathrm{l} / \mathrm{sec}$ (17 $600 \mathrm{ng} / \mathrm{l} / \mathrm{hour}$ ) (upper limit of reference range for age is $0.9(3130)$ ).

The heart failure improved after the hypertension had been controlled. Surgical repair or balloon dilatation of the stenosed right renal artery were considêred but were not thought to be technically feasible. Medical management, with a view to future surgical repair, was also considered but the blood pressure remained labile. Despite the risk of reducing glomerular filtration in the right kidney further, captopril was introduced to her regimen and this successfully controlled the blood pressure. After this, a diethylene triamine penta-acetic acid (DTPA) scan showed no function of the right, but normal function of the left, kidney. A right nephrectomy was carried out. There was no evidence of renal artery dysplasia on histological examination of the kidney. Her postoperative recovery was uneventful and four days later all antihypertensive drugs were withdrawn. Ten months after nephrectomy her weight had risen to the 25th centile and she remained well with a blood pressure of $80 / 60 \mathrm{~mm} \mathrm{Hg}$. The peripheral plasma renin activity and echocardiographic findings had returned to normal.

\section{Discussion}

All three patients presented with severe heart failure, a complication of sytemic hypertension in neonates and young children which is underdiagnosed. Angiography showed that stenosis of the renal arteries (main artery in cases 2 and 3, intrarenal arteries in case 1), was the cause of the hypertension.

Case 1 developed symptoms on the third day of life. The finding of cardiac ventricular hypertrophy indicated a chronic change, presumably as a result of fetal hypertension. It is likely that the tortuous dilation of the proximal arteries of the aortic arch were also caused by fetal hypertension rather than being the result of a generalised vascular disorder. There was no other evidence to suggest a vasculopathy in this patient. As far as we are aware, fetal hypertension presenting in a neonate has not been previously described. A renovascular cause for hypertension secondary to an abnormality of the left kidney was suggested by the findings on renal arteriography and by the grossly increased plasma renin activities, although the latter must be interpreted with caution in patients receiving hypotensive drugs and during general anaesthesia. ${ }^{1}$ Although histology of the left kidney confirmed the angiographic findings of intrarenal segmental arterial stenosis, it was not possible to define the aetiology of the vascular abnormality.

The most frequently reported causes of neonatal hypertension are stenosis of the proximal renal artery and renal artery thrombosis or embolisation after umbilical arterial catheterisation. ${ }^{2}$ Renal vein thrombosis has also been implicated as a cause of neonatal hypertension, ${ }^{3}$ and has been described as a complication of maternal diabetes mellitus. ${ }^{4}$ None of our patients had undergone catheterisation of the umbilical arteries and in case 1 (whose mother had diabetes mellitus) renal ultrasonography and arteriography excluded thrombosis of the renal vessels. Evans et al described a neonate with hypertension detected within 24 hours of birth, with associated renal vein thrombosis. ${ }^{3}$ Evidence was presented that suggested that the thrombosis had occurred antenatally, but not the hypertension. In a necropsy series of neonates with renal vein thrombosis, one infant who died at 48 hours of age had biventricular hypertrophy. ${ }^{3}$ No blood pressure recordings were given, but the cardiac hypertrophy may have been secondary to systemic hypertension.

Our second and third patients had failed to thrive because of hypertension. Case 2 had been investigated for over 12 months, but his blood pressure had not been recorded. Case 3 presented during an intercurrent infection when she became dehydrated and seriously ill, possibly as a result of further renal ischaemia secondary to 
impaired circulating blood volume or renal infarction. Both patients thrived once their hypertension was controlled. Failure to thrive and acute or chronic heart failure are commonly encountered in neonates and older children. Although hypertension is known to cause heart failure and failure to thrive, blood pressure is frequently not measured routinely in these children. A working party of the British Hypertension Society has recommended that sick children should have their blood pressure measured whenever a general medical examination is undertaken. ${ }^{5}$ It is clear that this should include children who are failing to thrive, and that earlier measurement of blood pressure in at least one of our patients (case 2) would have resulted in reduced morbidity. All three case emphasise the importance of the early detection and appropriate treatment of hypertension.

Each of our patients had unilateral renal arterial disease and was 'cured' by unilateral nephrectomy. Nephrectomy may be associated with operative or anaesthetic complications and results in some reduction in overall kidney function. It does not guarantee a cure of the hypertension, as subtle abnormalities may exist in the apparently normal kidney that could continue to drive the hypertension. Sometimes, however, the potential benefits outweigh the risks. A successful nephrectomy provides a definitive cure, and removes the need for life long drug treatment and the risk of further potentially serious episodes of hypertension that may be caused either by non-compliance with medication or by labile blood pressure.

Patients with bilateral intrarenal vascular disease have no alternative to life long drug treatment. In patients with unilateral intrarenal disease, however, the choice is between drug treatment and unilateral nephrectomy, the decision being based on a balance between the degree of functioning renal tissue that is lost, the amount of hypotensive medication that is required, and the difficulties with compliance with medication and monitoring of follow up. In case 1 unilateral nephrectomy resulted in the loss of less than a quarter of total kidney function and avoided the need for life long drug treatment.

Reconstructive vascular surgery or balloon dilatation may be feasible in patients with extrarenal arterial stenosis, but do not guarantee success. ${ }^{6}$ In case 2 the stenosis was extrarenal but was tight; this would have necessitated major reconstructive surgery to save the function of a small kidney, or long term medication with several drugs, or both. In case 3 the stenosis was also extrarenal, but the blood pressure was dangerously high until captopril was introduced into the regimen. This caused loss of function of the affected kidney, presumably as a result of a reduced glomerular filtration pressure, ${ }^{7}$ and consequently this kidney contributed nothing to total renal function. In this patient, therefore, nephrectomy did not result in any further loss of renal function.

The authors are grateful to Miss Sharon White for her help in preparing the manuscript.

1 Dillon MJ. Clinical aspects of hypertension. In: Holliday MA Barratt TM, Verner RL, eds Pediatric nephrology. MA, Barratt TM, Verner RL, eds. Pediatric nephrology

2 Adelman RD. The hypertensive neonate. Clin Perinatol 1988;15:567-85.

3 Evans DJ, Silverman M, Bowley NB. Congenital hypertension due to unilateral renal vein thrombosis. Arch Dis Child 1981;56:306-8.

4 Takeuchi A, Benirschke K. Renal venous thrombosis of the newborn and its relation to maternal diabetes. Biol Neonate 1961;3:237-56.

5 de Swiet M, Dillon M J, Littler W, O'Brien E, Padfield P L, Petrie J C. Measurement of blood pressure in children:
recommendations of a working party of the British Hypertension Society. Br Med f 1989;299:497.

6 Anonymous. Operating on renovascular hypertension [Editorial]. Lancet 1983;ii:1007-8.

7 Wenting G J, Ton-Tjiong HL, Derx FHM, de Bruyn JHB, Man in 't Veld AJ, Schalekamp MADH. Split renal function after captopril in unilateral renal artery stenosis. Br Med f 1984;288:886-90. 